\title{
Superior Hypogastric Plexus Block for Chronic Pelvic Pain Through an S1 Transforaminal Approach: A Case Report
}

Zahra Sykes, MD, Phillip Suwan, MD, Sachin Bahadur, MBBS, Daniel Atkinson, MD, Zhuo Sun, MD, and Anterpreet Dua, MBBS

Chronic prostatitis/chronic pelvic pain syndrome (CPPS) is a debilitating syndrome commonly seen in men under the age of 50 years, which greatly impacts the quality of life. The treatment is challenging, which often requires a multimodal management approach. The superior hypogastric plexus is located anterior to L5 and S1 vertebral bodies in the retroperitoneal space and contains afferent pain fibers from most of the pelvic structures. Performing a superior hypogastric plexus block (SHPB) can potentially alleviate pain originating from various pelvic regions and structures. It is currently a viable therapy for many syndromes including endometriosis, interstitial cystitis, irritable bowel syndrome, and pain after pelvic surgery.

In this case report, we present a patient who had chronic pelvic pain with a poor response to conservative management. Initially, attempts at an SHPB from the classic posterolateral approach were unsuccessful. This technique for performing this block can prove difficult due to vasculature variability or anatomic barriers, such as the iliac crest and transverse process of the fifth lumbar vertebrae. Thus, a left S1 transforaminal approach was used to block the plexus. This provided the patient with one month of near $100 \%$ pain relief, with gradual return to baseline thereafter.

CPPS poses unique treatment challenges. Although often treated conservatively, SHPB is a valid treatment option for those who fail to respond adequately to other modalities. An S1 transforaminal approach is a novel and valuable alternative technique for SHPB in patients with compromising anatomy.

Key words: Chronic prostatitis, chronic pelvic pain, superior hypogastric plexus, superior hypogastric plexus block, pelvic trauma, pelvic pain in men
Chronic prostatitis (CP)/chronic pelvic pain syndrome (CPPS) is a clinical syndrome defined on the basis of constant or recurring urologic symptoms and/or pain in the pelvic region lasting for 3 of the preceding 6 months in the absence of bacterial infection (1). With an estimated prevalence of $2 \%$ to $16 \%$ worldwide, it is a relatively common condition in men

From : Augusta University Medical College of Georgia, Augusta, GA

Author for correspondence: Zahra Sykes, MD

Address: Department of Anesthesia, Augusta University, 1120 15th St., 30912, Augusta, GA 48059-3118

E-mail: zsykes@augusta.edu under the age of 50 years, which can significantly impact the quality of life (2). Because of the wide constellation of symptoms in patients with CP/CPPS, it is a difficult condition to treat. At this time, there is no widely accepted gold standard in the treatment of CPPS, however, a number of pharmacologic and nonpharmacologic interventions have been attempted. Once conservative management fails to relieve the pain, interventional management should be considered.

The superior hypogastric plexus (SHP) is a plexus of nerves situated on the vertebral bodies anterior to the bifurcation of the abdominal aorta in the retroperitoneum. Pelvic afferent and efferent sympathetic nerves from branches of the aortic plexus and fibers from 
L2 through L3 splanchnic nerves form the SHP. This plexus innervates most pelvic structures including the descending colon, prostate, rectum, and internal genitalia, excluding the ovaries and fallopian tubes. The superior hypogastric plexus block (SHPB) for the treatment of pelvic cancer pain is well established. In addition, it has been used successfully in patients with endometriosis, interstitial cystitis, irritable bowel syndrome, and pain after pelvic surgery. Various approaches to this procedure have been reported, owing to the anatomic obstacles of a high iliac crest or large transverse process of the fifth lumbar vertebra. Here, we report a case using a novel S1 transforaminal approach after unsuccessful attempts with the conventional approach. As far as we know, this is the first time reporting an SHPB using the S1 transforaminal approach in literature.

\section{CASE DESCRIPTION}

An African American man aged 37 years with a history of hypertension presented to our pain center for evaluation of his chronic pelvic pain. He reported a trauma at the age of 17 years from falling onto a steel beam penetrating his rectum, prostate, and bladder. $\mathrm{He}$ underwent colostomy and ureterostomy with a colostomy reversal one year later.

Following the acute phase of his injury, he developed constant perineal and suprapubic pain along with a sensation of incomplete voiding. The pain, rated $10 / 10$, radiated to the base of his penis, testes, rectum, and prostate, and affected his ability to walk. Associated symptoms included difficulty voiding and bowel movements secondary to pain. His pain affected his ability to maintain a job and engage in intercourse. Attempts at pain control consisted of acetaminophen-hydrocodone and nonsteroidal antiinflammatory drugs that yielded minimal relief.

Magnetic resonance imaging of the pelvis with and without contrast medium revealed findings consistent with prostatitis, and no evidence to suggest prostatic abscess. He ultimately underwent a transurethral incision of the bladder neck, which improved his voiding symptoms. However, he continued to have chronic prostate and rectal pain, prompting his referral.

The patient was prescribed oral amitriptyline and gabapentin. At his 2-month follow-up visit, his gabapen- tin dose was incrementally titrated without significant improvement. Because of his lack of improvement, we proceeded with an SHPB.

In the fluoroscopy suite, the patient was placed in the prone position and prepped in the standard sterile fashion. A left $\mathrm{S} 1$ transforaminal approach to the SHP was planned after attempts at left and right L5/S1 paravertebral approaches led to intravascular contrast medium uptake. A 5-inch 22-gauge spinal needle was advanced toward the middle of the left S1 foramen under anterior-posterior and lateral fluoroscopic views. The needle was advanced until the tip was just anterior to the ventral margin of the foramen. Contrast medium injection (Omnipaque 240, $1 \mathrm{~mL}$ ) (GE Healthcare AS, Oslo, Norway) showed excellent spread, bilaterally, tracking cephalad toward the L5 level. There were no signs of washout or uptake. Due to sufficient bilateral spread of the contrast medium, a right-sided approach was deemed unnecessary. A combination of methylprednisolone $80 \mathrm{mg} / 1 \mathrm{~mL}$ and $12 \mathrm{~mL}$ of $0.25 \%$ bupivacaine was injected into the space. The patient tolerated the procedure well and reported an $80 \%$ reduction in pain prior to discharge. Images from this patient's SHPB were unavailable. However, fluoroscopic images illustrating the described technique are included. Notably, in these images we were unable to demonstrate the bilateral contrast medium spread achieved using the unilateral approach as in our case detailed earlier (Figs. 1 and 2).

At his 2-month follow-up visit, he reported nearly $100 \%$ pain relief for one month following the block, but with an eventual return to baseline. Given the positive diagnostic/therapeutic block, he was offered a repeat SHPB along with the use of radiofrequency ablation in an effort to provide prolonged relief. Also included in the treatment options was spinal cord stimulation (SCS) therapy. After full review of his therapeutic options, he opted to pursue a trial of SCS therapy.

\section{DISCUSSION}

CP/CPPS is a clinical syndrome defined on the basis of constant or recurring urologic symptoms and/or pain in the pelvic region lasting for 3 of the preceding 6 months in the absence of bacterial infection. Men with CP/CPPS present with pelvic pain in the absence of urinary tract infections. Symptoms result from an 
interplay of psychological factors, trauma, and the combined responses of the immune, neurologic, and endocrine systems (3). This manifests as pain in the perineum, rectum, prostate, penis, testicles, and abdomen. Although bacterial infections have been suspected in the inflammatory subset of $C P$, there appears to be little correlation between prostatic inflammation and the presence of CP/CPPS symptoms. Prostatic biopsy specimens obtained via transperineal approach from men with and without CP/CPPS have shown no differences in their culture results. Thus, despite multiple theories, the etiology of CP remains unknown (4). In patients with CPPS, a multimodal therapeutic approach is often necessary. Interventional procedures are often used in patients once conservative measures have failed. The most common and successful therapeutic approaches include alpha-adrenergic antagonists, 5-alpha reductase inhibitors, and psychosocial therapy. The use of alpha blockers as monotherapy has yielded mixed results in placebo-based trials. Dutasteride, a 5 -alpha reductase inhibitor, has reduced prostatitis symptoms when compared to placebo. Psychotherapy for patients and their spouses can help improve coping mechanisms. These therapeutic options include: progressive relaxation training, self-hypnosis, biofeedback, and cognitive behavioral therapy $(5,6)$.

Additional treatment options for patients include cannabis, which has been shown to suppress chronic inflammatory pain by stimulating cannabinoid receptors. In a joint Canadian study with the Prostatitis Foundation, authors studied men with CP/CPPS and their experience with cannabis. The majority of patients reported that cannabis made their mood, pain, and muscle spasms "slightly/much better." However, regular cannabis use has been associated with lower sperm concentration, lower sperm total count, and decreased sperm function. Because CP/CPPS is a condition seen in men younger than age 50 years, therapeutic cannabis use should be avoided by men who hope to have children in the future (7). Interventional options attempted in the past have included ultrasound-guided therapeutic options for pudendal neuralgia, piriformis syndrome, and ilioinguinal, iliohypogastric, and genitofemoral nerve neuropathy (8). Other alternative approaches to treatment have included acupuncture, Botox, and lower intensity pulsed ultrasound (9-11). Patients afflicted

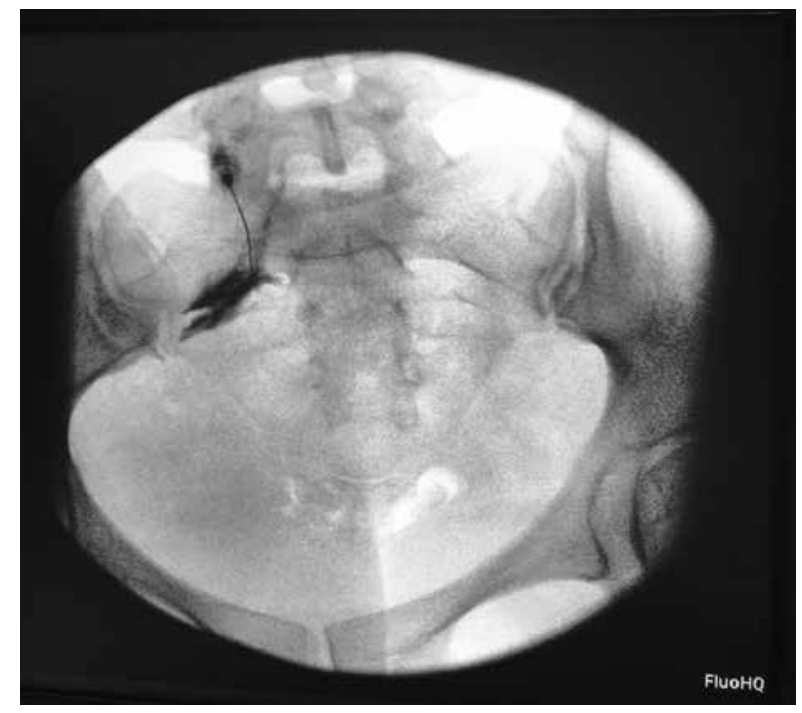

Fig. 1. Anterior-posterior view demonstrating needle placement through the $\mathrm{S} 1$ foramen and the corresponding contrast medium spread.

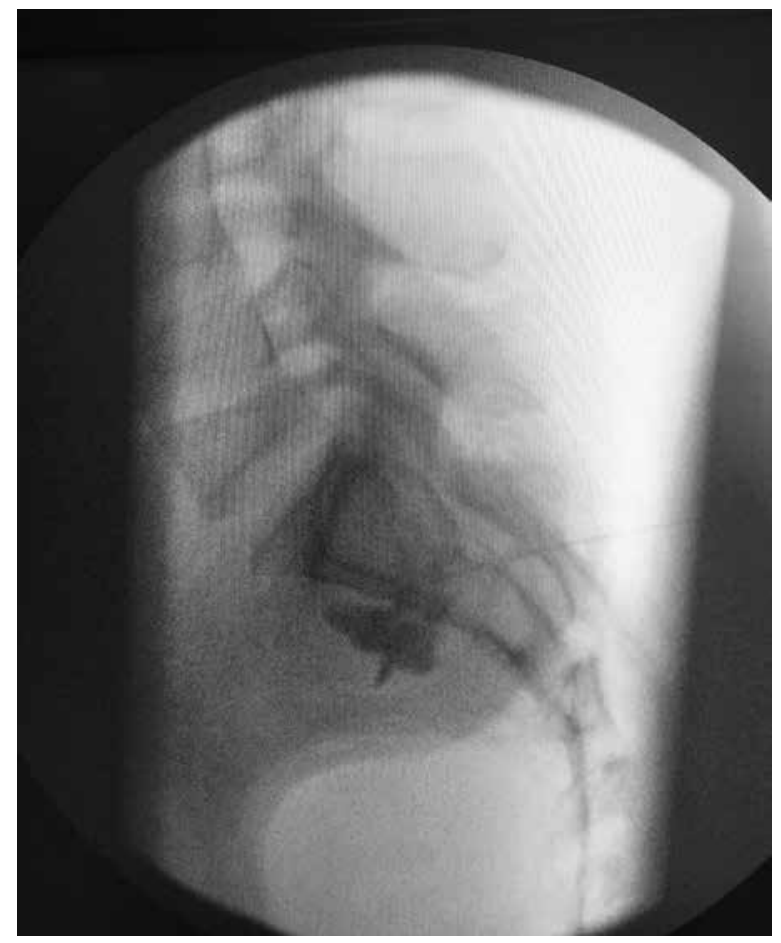

Fig. 2. Lateral view demonstrating S1 foramen approach to the superior hypogastric plexus and the corresponding spread of contrast medium. 
with CPPS have proved to be both diagnostically and therapeutically challenging.

The SHP is located below the aortic bifurcation and is an extension of the aortic plexus in the retroperitoneum. Pelvic afferent and efferent sympathetic nerves from branches of the aortic plexus and fibers from L2 through L3 splanchnic nerves form the SHP. This plexus innervates most pelvic structures including the descending colon, prostate, rectum, and internal genitalia, excluding the ovaries and fallopian tubes, which receive both sympathetic and parasympathetic innervation from the ovarian and uterine (pelvic) plexuses.

Since the SHPB was first introduced to treat pelvic cancer pain (12), it has been used successfully in patients with endometriosis, interstitial cystitis, irritable bowel syndrome, and pain after pelvic surgery. The use of the superior hypogastric block for CPPS, although not a standard of care, does have some precedent in the literature. Moreover, although most often performed using a posterolateral or a L5-S1 transdiscal approach, an S1 transforaminal approach as we demonstrated can also be safe and effective.

Michalek et al (13) described 2 cases about using SHBP to treat noncancer pelvic pain. The first case was a 43-year-old patient suffering from burning pain of the urethra without sufficient response to analgesics and antidepressants. The second case was a 68 -year-old man suffering from chronic burning and itching pain of the urethra and glans penis failed with conservative therapy (anti-inflammatory drugs, tramadol, spasmolytics). Both patients received SHPB. The visual analog scale scores (VAS) decreased from 6 and 5 prior to block, to 1 and 0 , respectively, 24 hours after the block (13). A single-center retrospective study described 22 patients with chronic pelvic pain who were treated with SHPBs from 1996 to 2000. The causes of pelvic pain in this group were diverse and included endometriosis, adhesions, interstitial cystitis, and postprostatectomy pain. In this study, 10 out of 22 patients had a positive response to diagnostic blockade, and 11 of these patient's underwent neurolysis. Out of these 11 patients, 4 patients had significant pain relief following the procedure, and one patient had complete resolution of pelvic pain. Although this study showed positive results for SHPB, it was a small retrospective study with patients who presented with diverse causes of chronic pelvic pain.

Since the introduction of the traditional SHPB in 1990 by Plancarte et al (14), various methods of approach have been attempted. The reason is due to anatomic barriers, such as the nerve root, iliac artery and vein, iliac crest, and transverse process of the L5 vertebra. In our case, we started with the classic posterolateral approach, but the needle was unable to safely reach the target area. We then attempted the S1 transforaminal approach with a satisfactory outcome. As far as we know, this is the first case to report an SHBP with S1 transforaminal approach in literature review. We understand that each of the current various techniques have advantages and disadvantages. For example, the disadvantages of the traditional method introduced by Plancarte et al (14) include the difficulty to avoid contact with the transverse process, and to position the needle in the accurate position. Walder's approach is performed under computed tomography guidance, which put patients under the extensive exposure to radiation (15). The anterior approach can avoid the barriers from the back, but put the colon, bladder, and surrounding vessels at risk. The transdiscal approach is another alternative route for SHPB, in which a successful block can be achieved with single needle injection (16). The possible complication of the method is the risk of discitis or infection. Our approach also has disadvantages. The sacral nerve plexus crosses the foramina from medial to lateral. The foraminal branch is provided by the lateral sacral artery and enters the inferior lateral quadrant of the foramen. Our approach may place these structures under the risk of injury during the injection, and therefore is an option in patients with anatomic difficulties.

\section{CONCLUSIONS}

CPPS is a common condition with a significant negative impact on the quality of life of patients. Once a multimodal conservative approach fails, the SHPB is an alternative option for pain control in these patients. The $\mathrm{S} 1$ transforaminal approach could be an alternative technique to perform SHPB in patients with anatomic barriers. Further studies are needed to assess the efficacy of this approach. 
Disclaimer: There was no external funding in the preparation of this manuscript.

Conflict of interest: Each author certifies that he or she, or a member of his or her immediate family, has no commercial association (i.e., consultancies, stock ownership, equity interest, patent/licensing arrangements, etc.) that might pose a conflict of interest in connection with the submitted manuscript.

\section{REFERENCES}

1. De Leon-Casasola OA, Kent E, Lema MJ. Neurolytic superior hypogastric plexus block for chronic pelvic pain associated with cancer. Pain 1993; 54:145-151.

2. Smith $\mathrm{CP}$. Male chronic pelvic pain: An update. Indian J Urol 2016; 32:34-39.

3. Schaeffer AJ. Chronic prostatitis and the chronic pelvic pain syndrome. N Engl J Med 2006; 355:1690-1698.

4. Lee JC, Muller $\mathrm{CH}$, Rothman I, et al. Prostate biopsy culture findings of men with chronic pelvic pain syndrome do not differ from those of healthy controls. J Urol 2003; 169:584-588.

5. Pontari MA, Ruggieri MR. Mechanisms in prostatitis/chronic pelvic pain syndrome. J Urol 2004; 172:839-845.

6. Krieger JN, Nyberg L Jr, Nickel JC. NIH consensus definition and classification of prostatitis. JAMA 1999; 282:236-237.

7. Wei X, Wu X, Lovinger D, Zhang L. A common molecular basis for exogenous and endogenous cannabinoid potentiation of glycine receptors. J Neurosci 2012; 32:5200-5208.

8. Sandhu J, Tu HYV. Recent advances in managing chronic prostatitis/chronic pelvic pain syndrome. F1oooRes 2017; 6:F1000 Faculty Rev-1747.

9. Li HS, Wang B, Han L, Wang CH, Xin ZC. Transperineal ultrasonic therapy for chronic prostatitis. Zhonghua Nan Ke Xue 2013; 19:49-53.

10. Blanchet KD. Acupuncture: Gaining acceptance in urolo-

gy: Proves effective for a number of chronic conditions. B]U Int 2012; 109:ii-iv.

11. Falahatkar S, Shahab E, Gholamjani MK, Kazemnezhad E. Transurethral intraprostatic injection of botulinum neurotoxin type A for the treatment of chronic prostatitis/chronic pelvic pain syndrome: Results of a prospective pilot double-blind and randomized placebo-controlled study. BJU Int 2015; 116:641-649.

12. Mishra S, Bhatnagar S, Gupta D, Thulkar S. Anterior ultrasoundguided superior hypogastric plexus neurolysis in pelvic cancer pain. Anaesth Intensive Care 2008; 36:732-735.

13. Michalek P, Dutka J. Computed tomography-guided anterior approach to the superior hypogastric plexus for noncancer pelvic pain: A report of two cases. Clin J Pain 2005; 21:553-556.

14. Plancarte R, Amescua C, Patt RB, Aldrete JA. Superior hypogastric plexus block for pelvic cancer pain. Anesthesiology 1990; 73:236-239.

15. Waldman SD, Wilson WL, Kreps RD. Superior hypogastric plexus block using a single needle and computed tomography guidance: Description of a modified technique. Reg Anesth 1991;16:286-287.

16. Liliang PC, Hung CM, Lu K, Chen HJ. Fluoroscopically-guided superior hypogastric plexus neurolysis using a single needle: A modified technique for a posterolateral transdiscal approach. Pain Physician 2018; 21:E341-E345. 
